REVISTA DE LA CEPAL 83 . AGOSTO 2004

\title{
Seguridad alimentaria y agricultura familiar
}

\author{
Gustavo Gordillo de Anda
}

$\mathrm{E}$

ste trabajo parte del debate sobre la seguridad alimentaria que ha tenido lugar después de la Cumbre Mundial sobre la Alimentación realizada en 1996. Se analiza la viabilidad de un tipo de propuesta programática que deriva de la Declaración de Roma y del Plan de Acción suscritos por los gobiernos de 148 países, y se sugiere que para llevarlo a cabo se requiere de un vínculo estructural entre la población que se encuentra en condiciones de inseguridad alimentaria, generalmente desprovista de voz y de capacidad de presión, y las diversas instancias encargadas de impulsar intervenciones públicas. Se plantea como hipótesis central de este trabajo que ese vínculo estructural puede construirse a partir de los agricultores familiares. Y en torno al derecho a la alimentación, se postula que la seguridad alimentaria sustentada en ese derecho es una expresión territorial de derechos ciudadanos.

Gustavo Gordillo de Anda Subdirector General de la Organización de las Naciones Unidas para la Agricultura y la Alimentación (FAO) Representante Regional para América Latina y el Caribe

๑ gustavo.gordillodeanda@fao.org 


\section{Seguridad alimentaria: nuevas visiones} y nuevas prácticas

Aunque, como hoy sabemos, el mundo produce muchos más alimentos de los que se necesitan para atender las necesidades de sus habitantes, casi 800 millones de seres humanos — uno de cada siete- sufren hambre (FAO, 2002a). La superación de este flagelo no pasa tanto por aumentar la producción agrícola en los países en desarrollo, como antiguamente se creía, sino por generar oportunidades de empleo e ingresos, así como por ampliar la posibilidad de comercializar los productos agrícolas generados por la población de estos países.

Los últimos 50 años de la agricultura familiar han estado marcados por una combinación de: aumento de los precios de los insumos y aperos para la producción agrícola, disminución de los subsidios y del aparato protector estatal y una caída vertiginosa en los precios de mercado de los productos agrícolas.

Esa caída se ha debido a las transformaciones en las telecomunicaciones y la computación, la revolución verde y los cambios en materia de biotecnología. En la actualidad, sin embargo, la causa principal parece hallarse en las políticas de subsidios a la producción y a la exportación que aplican los países desarrollados, especialmente los de la Organización de Cooperación y Desarrollo Económicos (OCDE). Un estudio del Departamento de Agricultura de los Estados Unidos (Burfisher, 2001) encontró que los aranceles y subsidios de los países desarrollados deprimen los precios agrícolas hasta en un $12 \%$ y que originan casi el $80 \%$ de las distorsiones del comercio mundial.

En conjunto, la distorsión de la capacidad exportadora de los países en desarrollo y la caída de los precios agrícolas internacionales estarían contribuyendo de manera decisiva a un menor desarrollo del sector agrícola en estos países (Rello y Trápaga, 2001).

No obstante, según Burfisher (2001), para eliminar las distorsiones a la capacidad exportadora de los

$\square$ Las informaciones y puntos de vista que contiene este artículo son de exclusiva responsabilidad de su autor y no coinciden necesariamente con los de la FAO. El autor agradece a Hernán Gómez, Carlos Icaza, Paul Lewin y Rodrigo Paillacar sus comentarios y colaboración en la edición preliminar de este artículo. países en desarrollo no bastaría con suprimir los aranceles y subsidios a la producción y exportación de los países desarrollados. Esas medidas tendrían que combinarse con otras destinadas a mejorar en los países en desarrollo la competitividad de sus economías agrícolas, y en especial de sus pequeños agricultores, sobre todo a través de inversiones en infraestructura y formación de capital humano; a fortalecer sus instituciones, y no menos importante, a superar los altos niveles de desigualdad social, pobreza y subnutrición que los aquejan.

\section{La situación en América Latina y el Caribe}

Como se ha venido señalando, el tema de la seguridad alimentaria mundial en el corto plazo no es estrictamente un problema técnico. Es un problema de carencia de medios de producción para satisfacer la demanda de alimentos, así como de falta de poder adquisitivo de los grupos más necesitados de las zonas rurales y urbanas (FAO, 2000). En América Latina y el Caribe existen casi 54 millones de personas que sufren hambre y desnutrición. Lejos de disminuir, este número se ha elevado en algunas subregiones, siendo especialmente preocupante la situación de Centroamérica y el Caribe. Además, hay en la región aproximadamente 211 millones de pobres, 11 millones más que en 1990, de los cuales 89 millones viven en extrema pobreza. La mayor incidencia de pobreza (CEPAL, 2002) se encuentra en el sector rural; allí casi el 54\% de la población se halla bajo la línea de pobreza, y el 31\% está bajo la línea de indigencia, es decir, con su ingreso no puede satisfacer sus necesidades alimentarias básicas.

Los problemas de inseguridad alimentaria y pobreza se ven agravados por la desigualdad en la distribución de los ingresos. En la mayoría de los países de la región el 10\% de los hogares más ricos percibe más del $30 \%$ de los ingresos, en tanto que la fracción de ingresos recibida por el $40 \%$ de los hogares más pobres se ubica, en casi todos los países de la región, entre el 9\% y el 15\% (CEPAL, 2002). Producto de los bajos salarios locales, los niveles de producción y productividad conseguidos han permitido a algunos países convertirse en 
exportadores de algunos productos agrícolas y aumentar sus ingresos. Sin embargo, lo cierto es que la pobreza extrema y la subnutrición crónica no han desaparecido y en muchos países han aumentado (FAO, 2002a).

En suma, las transformaciones agrícolas del último medio siglo han llevado a la agricultura de la región hacia dos extremos: por un lado se halla una agricultura moderna, rentable, mecanizada, que utiliza productos agroquímicos y variedades de alto rendimiento, y por el otro, una agricultura de subsistencia, empobrecida, excluida y hambrienta (FAO, 2000).

\section{La faO y el Programa Especial de Seguridad Alimentaria}

Con las metas de la Cumbre Mundial sobre la Alimentación en mente, la Organización de las Naciones Unidas para la Agricultura y la Alimentación (FAO) ha impulsado el Programa Especial de Seguridad Alimentaria (PESA). Éste se compone de acciones tendientes a mejorar la seguridad alimentaria de un país y pretende ser algo más que un proyecto demostrativo, pues busca proponer y desarrollar políticas nacionales de seguridad alimentaria en el país que lo solicite. En su concepción inicial, partió de la premisa de que existen tecnologías viables que, al ser aplicadas correctamente en los países más afectados por problemas de seguridad alimentaria, podrían incrementar la productividad de la agricultura y la producción de alimentos. La visión original estaba demasiado orientada al ámbito de la oferta de alimentos y no consideraba el papel de las instituciones y de los actores en los resultados que se obtendrían.

Las tendencias que exhiben actualmente las dimensiones económicas, políticas y sociales ${ }^{1}$ han llevado a una nueva concepción de la seguridad alimentaria $\mathrm{y}$, con ello, a la necesidad de que la FAO reoriente sus programas en esta materia. Efectivamente, las evaluaciones realizadas al PESA han subrayado lo siguiente (FAO, 2002c):

\footnotetext{
${ }^{1}$ Entre otras, caída de los precios internacionales; altos niveles de pobreza, hambre, subnutrición e inequidad; heterogeneidad en la dotación de activos de los agricultores familiares; segmentación de los mercados laborales; bajos niveles de inversión de la familia rural; asimetría de la información en los mercados; escasa disponibilidad y mala asignación de bienes públicos en el sector rural; dualidad del sector rural en que coexisten dos sectores, uno competitivo y otro de subsistencia; falta de infraestructura rural; falta de capacidad institucional; poca capacidad de respuesta de los sistemas de investigación y desarrollo y existencia de una brecha digital entre las zonas rurales y urbanas.
}

i) “Cuando comenzó a ejecutarse, el PESA había sido formulado con arreglo a lo que el Equipo de evaluación considera un diseño rígido e inflexible. Se determinó también que inicialmente se pondría en práctica en zonas que tenían el potencial de aumentar rápidamente la producción, lo que significaba que en esas zonas debían existir posibilidades de riego. Se consideraba que centrar las iniciativas en la producción contribuiría a resolver los problemas de seguridad alimentaria tanto en los hogares como a nivel nacional. Pronto quedó patente que el enfoque inicial a nivel "micro", orientado a la producción, era insuficiente para garantizar el progreso en la solución del problema de la seguridad alimentaria y que las cuestiones de tipo "meso" y "macro" eran importantes para conseguir aumentos de la producción $\mathrm{y}$ asegurar que los productores obtuvieran beneficios".

ii) Como consecuencia de lo anterior, "los lugares seleccionados para realizar las actividades del PESA en los países estudiados presentan en general una productividad relativamente elevada en comparación con las zonas más marginales, en las que la malnutrición de las zonas rurales es mayor y el potencial para aumentar la productividad agrícola más reducido. Así pues, aunque a juicio del Equipo de evaluación las zonas elegidas para las actividades del PESA son, probablemente, las que presentan mejores condiciones desde el punto de vista de la mejora de la seguridad alimentaria nacional, el impacto de las actividades del PESA en la mejora de la seguridad alimentaria familiar habría sido mayor en las zonas marginales".

iii) "El éxito de intervenciones como el PESA depende en gran medida de la solidez de las estructuras institucionales, incluidos los sistemas de extensión, crédito, distribución de insumos y comercialización de los productos. Cuando existen deficiencias en estos aspectos, es muy poco probable que un plazo de dos o tres años sea suficiente para que se puedan apreciar efectos notables".

iv) "No fue posible disponer de datos sistemáticos sobre el grado de adopción de las tecnologías demostradas en el marco del PESA, tanto porque no se ha recogido esa información, como porque muchos de los proyectos están todavía en curso de ejecución o han concluido en fecha reciente. [...] se encontraron pocas pruebas de que siguieran utilizándose las tecnologías después de las demostraciones de los proyectos o de que las 
adoptaran campesinos que no habían tenido relación con el PESA".

v) "En el marco del PESA se conceden frecuentemente subvenciones para fomentar la adopción de tecnologías. Esto suele hacerse de dos formas distintas: proporcionando gratuitamente insumos a los agricultores y aportándolos a precios subvencionados. Estos procedimientos deben ser revisados porque no favorecen la sostenibilidad de las tecnologías una vez que han concluido las ayudas directas a las iniciativas".

vi) "En general, el PESA ha tenido hasta la fecha efectos limitados sobre las políticas nacionales relativas a la seguridad alimentaria y sobre la comunidad de donantes en cuanto a las estrategias destinadas a aumentar la seguridad alimentaria en los países de bajos ingresos y con déficit de alimentos (PBIDA) y la movilización de recursos para la continuación del programa....".

El PESA también ha experimentado adaptaciones específicas a las condiciones prevalecientes en América Latina y el Caribe: i) se financia con recursos públicos nacionales; ii) se da en países de desarrollo intermedio ${ }^{3}$ y no sólo en aquellos de bajos ingresos y con déficit alimentario; iii) es multisectorial; iv) tiene cobertura nacional desde el inicio, y v) es de larga duración. Asimismo, algunos fenómenos propios de la región, o de algunas zonas dentro de ella, afectan la seguridad alimentaria; entre esos fenómenos están la debilidad institucional; los problemas de consolidación de los procesos descentralizadores; la alta vulnerabilidad a desastres naturales y la imperiosa necesidad de mejorar el manejo de los riesgos (por ejemplo, a través de sistemas de alerta temprana); el alto riesgo social y económico de depender excesivamente de algún producto básico que puede sufrir caídas dramáticas,

\footnotetext{
${ }^{2}$ La evaluación la llevó a cabo un equipo representativo de nueve consultores externos superiores con el apoyo operativo del Servicio de Evaluación de la FAO. El Equipo de evaluación visitó las oficinas regionales de la FAO y 12 países provenientes de las regiones en desarrollo en las que se ha aplicado el PESA (a saber, Bangladesh, Bolivia, Camboya, China, Ecuador, Eritrea, Haití, Mauritania, Níger, Senegal, Tanzania y Zambia). El Equipo de evaluación seleccionó los países que debería visitar, basándose en una lista preparada por la FAO de 18 países, representantes de todas las regiones en desarrollo. En esos países, el trabajo sobre el terreno se había iniciado al menos tres años antes y había abarcado al menos tres de los cuatro componentes del PESA. Los criterios de selección permitieron tener la certeza de que el Equipo efectuaría la labor de evaluación sobre la base de una experiencia considerable en la aplicación del PESA.

${ }^{3}$ Tal sería el caso de Brasil, México, la República Dominicana y Venezuela.
}

como el café; y las diversas tensiones que provocan los procesos de integración comercial —como el que apunta al Área de Libre Comercio de las Américas-y los tratados.

En esta visión actualizada de la seguridad alimentaria, concebida como un derecho de las personas y cuyo objetivo central es mejorar la capacidad de las familias para acceder a los alimentos, la unidad de referencia deja de ser la finca y pasa a ser la familia rural. Esto genera una concepción integral, orientada a mejorar el nivel de bienestar de las personas más que a incrementar la producción o la productividad agrícolas.

Así, la seguridad alimentaria deja de ser concebida como un simple ejercicio de transferencia tecnológica para establecerse como un derecho ciudadano. De aquí surge una pregunta crucial: ¿a qué segmento de la población están dirigidos estos programas de seguridad alimentaria?

Evidentemente, toda política de seguridad alimentaria que hace del derecho a la alimentación su principal eje discursivo requiere una clara definición de la población vulnerable, como los campesinos sin tierra, los pequeños agricultores, las familias campesinas, las poblaciones indígenas, las mujeres, los niños y los pobladores de zonas periurbanas. Sin embargo, el hecho de focalizar la población vulnerable no es en sí garantía de que esa población será incorporada en los programas y proyectos de seguridad alimentaria. Se precisa un vínculo estructural entre esa población, generalmente desprovista de voz y de capacidad de presión, y las diversas instancias encargadas de impulsar intervenciones públicas. Sin ese vínculo estructural, el vacío suele llenarse mediante el clientelismo político, o bien las intervenciones no llegan a la población vulnerable y son atrapadas por otros sectores con más poder de negociación. En este artículo se sugiere que dicho vínculo estructural puede construirse a partir de los agricultores familiares, tanto por su dinamismo como por su vasta presencia debida al carácter multifuncional de sus actividades productivas.

El interrogante central después de presentar el tipo de propuesta programática a la que llevan las versiones ampliadas de los programas de seguridad alimentaria es el siguiente: ¿qué grado de viabilidad política y económica tiene ese tipo de propuesta en el ámbito latinoamericano? Es claro que una estrategia básica para impulsar nuevas formas públicas de intervención en el medio rural supone la construcción de consensos acerca de los objetivos, términos y costos de las principales políticas para la promoción rural, y 
la conversión de estos acuerdos en reformas legales que garanticen el cumplimiento de los compromisos y la certidumbre de la continuidad de las políticas. Para poder juzgar la viabilidad de esta propuesta es necesario ahora examinar las condiciones que harían posibles los consensos. Comienzo con una reflexión sobre las movilizaciones sociales recientes en el medio rural y sobre la naturaleza de las reformas estructurales del decenio de 1990, que en buena medida están en el origen de esas movilizaciones, para después avanzar en un conjunto de propuestas específicas a partir del concepto de derecho a la alimentación.

\section{II}

\section{La movilización social como medio}

\section{de generar instituciones}

\section{Conjeturas sobre el sentido de las movilizaciones rurales}

Las profundas transformaciones que está experimentando América Latina hacen inevitables las protestas y la intranquilidad sociales. De acuerdo con el enfoque adoptado en este trabajo, las protestas sociales pueden ser vistas no sólo como una forma de cambiar la correlación de fuerzas y aumentar las capacidades negociadoras de ciertos actores sociales, sino también como un factor que induce a la reestructuración productiva y a la innovación institucional en sociedades bastante desiguales, fragmentadas y frágiles desde el punto de vista institucional. En esta perspectiva se tiene un dilema específico: ¿cómo dar solución a los conflictos sociales en el campo, evitando enfrentamientos irreversibles entre actores sociales? Y a la vez, ¿cómo aprovechar el impulso y el proceso de solución de conflictos para reforzar o generar nuevos acuerdos institucionales?

La movilización social surge como un proceso de posicionamiento ante los cambios de actores y organizaciones sociales que se sienten afectados, o potencialmente afectados, por la aparición de nuevos arreglos institucionales o por el abandono de otros antiguos. Aquí es necesario subrayar que todo intento de tipificar las movilizaciones rurales en categorías excluyentes tropieza con el hecho de que a menudo existe un traslapo de demandas y tipos de lucha, dado el carácter ambivalente y la naturaleza cambiante de los actores rurales en sus relaciones con la sociedad en general y con el Estado y el mercado en particular.

Es posible canalizar la movilización social mediante acuerdos basados en una ética de la responsabilidad que permita experimentar con arreglos institucionales innovadores. Evidentemente, no toda movilización social se convierte en innovación institucional, pues tiene en sí un alto riesgo disruptivo, que en América Latina es acicateado por dos factores cuya confluencia ha influido en el panorama actual de la región: una desigualdad social estructural —que nos viene de muy lejos y no sólo del período reciente de reformas- y procesos vertiginosos de cambio, llevados a cabo con prisa y descuido, que erosionan la cohesión social e impactan las certidumbres culturales.

Después de casi dos décadas de reformas estructurales, debiera quedar claro que cualquier sociedad es una construcción humana muy dinámica, en la cual los conflictos y las tensiones no son la excepción, sino la regla. Es todavía más evidente que estos conflictos se hacen presente en momentos como los actuales, cuando las sociedades están inmersas en profundos cambios de factura mundial y local. Justo en estas coyunturas es esencial establecer un vínculo fuerte entre un marco legal sólido y las movilizaciones sociales, con el propósito de ayudar al tránsito desde una protesta social - que por su propia naturaleza es espontánea y disruptiva - a una acción colectiva ciudadana. Este tránsito de la espontaneidad al sentido racional en la acción colectiva no sólo es un paso del plazo corto al largo en el horizonte de los actores sociales. Es también la base de los acuerdos mutuos entre los órganos del Estado y los actores sociales. Más importante aún, este puente entre legalidad y movilización puede garantizar la transformación de las protestas y conflictos sociales en innovaciones, experimentos y soluciones, tanto en el ámbito de las propias reglas para canalizar conflictos como en los ámbitos que originaron la movilización. Al proceso de construcción de estos puentes lo denomino movilización social como medio de generar instituciones. 
Lo que ha ocurrido recientemente en muchas de las movilizaciones sociales ocurridas en la región ha sido la irrupción espontánea y violenta de sectores de la sociedad, seguida por la represión directa, y por soluciones ad hoc y cortoplacistas para contener las movilizaciones. Véanse si no todos los casos en que las protestas han conducido a la renuncia de presidentes electos democráticamente: De la Rúa en Argentina, Fujimori en Perú y recientemente Sánchez de Losada en Bolivia. ${ }^{4}$

El mensaje de las recientes movilizaciones rurales parece relativamente claro, pese a ser ellas diversas y de distinta intensidad. Se reclama no sólo mayor igualdad de oportunidades, sino que además se rechaza una visión de justicia redistributiva centrada exclusivamente en acuerdos corporativos. Se demanda, además, participación en la toma de decisiones y en su puesta en marcha. El principio unificador es una visión ampliada de la noción de soberanía popular, en la cual el principio de justicia se articula con el principio de democracia. La libertad por la que se lucha implica igual participación en las diversas instancias de la sociedad y del Estado.

Frente a estas demandas, las reformas tendientes a la liberalización económica y política en América Latina habían prometido anular formalmente las políticas con sesgo antiagrícola; sin embargo, la apreciación del tipo de cambio, la caída sistemática de los precios agrícolas internacionales y la astringencia crediticia han anulado en buena parte las ventajas que para el sector agrícola había pregonado este modelo. En consecuencia, las políticas públicas rurales fueron aprovechadas por un pequeño sector oligárquico, que sesgó su elaboración e implementación, o bien subordinadas a los intereses de los actores urbanos más poderosos, tendiendo a ser accesorios de las estrategias de desarrollo.

\section{La ansiedad por concluir: ${ }^{5}$ el ritmo de las refor- mas}

La evolución del sector rural en América Latina en los últimos años puede describirse como una larga transición hacia formas institucionalizadas de gobernabilidad democrática.

\footnotetext{
${ }^{4}$ Lo que Luis Maira ha llamado "la revocatoria social de los mandatos presidenciales".

${ }^{5}$ Me refiero a la famosa frase de Flaubert, citada por Albert Hirschman en su importantísima obra sobre los esfuerzos reformistas inconclusos en América Latina en la segunda mitad del siglo pasado, titulada Journeys towards Progress (Hirschman, 1963).
}

Las reformas estructurales de las décadas recientes buscaban la estabilización económica como medio para lograr competitividad. Los principales cambios emprendidos fueron: la desregulación, la modernización de las burocracias y la privatización de las principales empresas estatales. ${ }^{6}$

Estas reformas dieron paso a un período de crisis institucional en el sector rural, marcado por cuatro factores: i) vacío institucional, ya que la liberalización económica se vio afectada por procesos de transferencia fallida o inconclusa de las funciones operativas del Estado hacia el sector privado en determinadas áreas, como la extensión agrícola, la comercialización de productos agropecuarios o el crédito formal; ${ }^{7}$ ii) desequilibrio entre la intención y la capacidad de renovación de las instituciones rurales; iii) resistencia al cambio y a las reformas, manifestada en la ausencia de mecanismos de diálogo y concertación, y iv) ausencia de sincronía entre el desarrollo institucional del sector rural y los cambios en el resto de la economía y de la sociedad.

La combinación de estos factores con frecuencia desembocó en una modernización selectiva en algunos sectores o regiones, con base en criterios económicos simplistas que clasificaron a las regiones, e incluso a las personas, como "viables" y "no viables". De hecho, éste fue un camino seguro hacia la profundización de los desequilibrios productivos y sociales en el sector rural.

Por su parte, el factor de eficiencia en la transformación institucional no puede ser separado de los factores distributivos involucrados en todos los tipos de reformas institucionales, que suponen cambios en el poder y en los procesos políticos.

En las condiciones actuales, el entorno macroeconómico de los países de la región no puede ofrecer por sí solo una alternativa real y duradera a los actores o

\footnotetext{
${ }^{6}$ Una primera fase buscó la estabilización económica, ajustando los precios macroeconómicos (tipo de cambio, tasa de interés y control de la inflación) para corregir los sesgos contra ciertos sectores y desatar crecimiento económico no inflacionario. Una segunda fase enfrentó las fallas de mercado a través del "cambio estructural"; sus principales ingredientes (liberalización comercial, privatizaciones y desregulación) pretendían mejorar y transparentar el financiamiento de los mercados para resolver los problemas de información asimétrica. Una tercera fase, que comienza apenas en muchos países de la región, apunta a las fallas de cooperación entre actores sociales (véase Gordillo, 1999).

${ }^{7}$ Los vacíos institucionales dejados por el retiro de algunas intervenciones - en ausencia de una política deliberada para la creación de nuevos arreglos institucionales - han sido ocupados desordenadamente por mercados paralelos y arreglos informales, a un costo económico y social alto, sobre todo cuando se analizan esos procesos a más largo plazo.
} 
zonas rurales desplazados. Por esto, el resultado seguro de la falta de políticas de desarrollo activas es el vacío institucional y la exclusión social.

En consecuencia, no tiene sentido promulgar la flexibilidad, la transparencia y la participación sin reconocer la importancia crucial de la intervención pública. A la vez, parece trascendental incorporar las numerosas estrategias diferenciadas y los actores sociales que las encarnan en un diálogo amplio que tenga como resultado la inclusión: la piedra angular de una estrategia efectiva de reformas rurales es la incorporación de todos los actores sociales dentro de los acuerdos institucionales que se estén gestionando, es decir, la inclusión de los trabajadores rurales, la agricultura familiar, las poblaciones indígenas, las mujeres, los agricultores comerciales, los empresarios agroindustriales, los inversionistas, y otros actores que están adquiriendo una importancia creciente, como las redes de supermercados. ${ }^{8}$

Pero este diálogo requiere reglas precisas y una estructura de incentivos para garantizar una participación sin exclusiones en el marco de una ética de la responsabilidad.

En tal contexto, entiendo por reconstrucción institucional un proceso deliberado emprendido por sectores mayoritarios del Estado y de la sociedad como respuesta a una situación límite — producto de una combinación de fragmentación social y debilidad institucional - que requiere el establecimiento de compromisos mutuos de derechos y obligaciones. A estos compromisos los denomino pacto de garantías, que es una construcción institucional específica a esas situaciones y que abarca los tres ámbitos institucionales: operativo, gubernativo y constitucional. Puesto que el pacto de garantías no se puede dar en el vacío, el papel de los actores sociales y sus movilizaciones tiene que estar ensamblado en este andamiaje institucional. El pacto de garantías tiene un propósito único: construir un piso básico para resolver diferencias inevitables en sociedades pluralistas. No se trata de mitificar el papel de la construcción de consensos, pero sí de señalar que es insustituible para modular el ritmo de cambio. Este proceso de reconstrucción institucional - cuya expresión es el pacto de garantías - puede ser alimentado por impulsos provenientes de la movilización social,

\footnotetext{
${ }^{8}$ Las políticas sectoriales ya no son capaces de atender los intereses de la mayoría de las familias dedicadas a la sola agricultura. Hoy se requiere un acercamiento a políticas territoriales que se extienden más allá de la agricultura para reducir las desigualdades y para explotar racionalmente los recursos naturales.
}

de determinadas políticas y de una buena estructura de incentivos y sanciones que garantice el cumplimiento de las reglas (enforcement institutions).

La reconstrucción institucional busca enfrentar problemas de cohesión social. Este debe ser el objetivo central de las políticas públicas en el sector rural, y debe tener una legitimidad social incontestable, que sólo puede proporcionar la participación de los actores rurales en el diseño e implementación de tales políticas.

Los actores rurales se interrelacionan con el cambio institucional: son agentes activos ${ }^{9} \mathrm{y}$ al mismo tiempo pueden ser sujetos afectados por la transformación. No todos los actores participan en la misma forma o al mismo tiempo en el cambio institucional, pero todos sienten sus repercusiones, aunque con diferente intensidad. Sin duda, la reconstrucción institucional en el ámbito rural puede significar que determinados agentes enfrenten el riesgo de que su existencia sea cuestionada, ${ }^{10} \mathrm{o}$ que tengan que adoptar una identidad colectiva diferente. Pero en todo caso lo fundamental es a qué ritmo han de llevarse a cabo esos cambios.

Desde el punto de vista de la economía política de las reformas - de su adopción y puesta en marcha- ${ }^{11}$ una consecuencia importante del enfoque que propongo en este texto es que la única forma de definir un "ritmo de cambio" que logre administrar la inestabilidad que conlleva, es a través de la construcción de consensos. El propósito es establecer una ruta de transformación que refleje el consenso, la direccionalidad y la certidumbre en las políticas agrícolas y rurales, con el fin de generar seguridad y evitar riesgos de acciones discrecionales y políticas agrícolas y rurales autoritarias. Estos elementos establecen una base fundamental para que el desarrollo institucional futuro contenga las características deseadas de autonomía, inclusión y acción subsidiaria efectiva por parte del Estado.

Es necesario, pues, visualizar un marco institucional que abarque no sólo las formas de intervención estatal o de organización de las agencias estatales, sino

\footnotetext{
${ }_{9}^{9}$ Participan o promueven el cambio institucional, por ejemplo, a través de movilizaciones; o allí donde existen foros o consejos locales (es decir, donde la interacción social está institucionalizada), mediante su participación en las discusiones o el proceso de toma de decisiones.

${ }^{10}$ Un cacique local, o un actor público ineficiente o no competente, podrá ser destituido o fiscalizado como resultado de un proceso de cambio institucional.

${ }^{11}$ Probablemente uno de los textos más destacados sobre el tema del ritmo de cambio sea la obra de Karl Polanyi, titulada The Great Transformation: The Political and Economic Origins of Our Time (Polanyi, 1957).
} 
también el conjunto de reglas y convenciones no formales e incluso incorpore normas éticas y morales de comportamiento que son parte de la interacción social. En este enfoque más amplio, el principal papel del desarrollo institucional es aumentar la eficiencia y reducir la incertidumbre mediante el diseño de una estructura estable, aunque no inmutable, que favorezca la interacción económica y social. Esa estructura de oportunidades e incentivos es el factor determinante del desempeño económico.

Dicho marco institucional trascendente, que emerge de estos cambios desde abajo y desde arriba, invita a un conjunto de reflexiones acerca del derecho a la alimentación como expresión territorial de derechos ciudadanos, y de la descentralización en el marco de una política de desarrollo regional.

\section{III}

\section{EI derecho a la alimentación y la descentralización}

\section{Avances en el enfoque del desarrollo basado en los derechos humanos}

Tanto el diseño de políticas públicas para favorecer la competitividad de los agricultores familiares como el establecimiento de reglas equitativas que permitan el acceso de sus productos al comercio internacional deben trascender la idea de que el desarrollo de los pueblos o de las personas y el combate al hambre son concesiones graciosas de la autoridad, y dejar establecido que, por el contrario, son parte de la obligación de todo Estado de garantizar derechos humanos universales a sus ciudadanos.

Aunque tradicionalmente los derechos humanos se han asociado sobre todo con el ámbito civil y político, vale la pena subrayar que a partir de la Declaración Universal de Derechos Humanos de 1948 se han reconocido con igual jerarquía diversos derechos económicos, sociales y culturales, que en 1976 dieron origen al Pacto Internacional de Derechos Económicos, Sociales y Culturales. En dicho Pacto se estableció la obligación de los Estados de garantizar el derecho a la alimentación, el cual se considera cumplido cuando las personas tienen acceso físico y económico, en todo momento, a la alimentación adecuada y a los medios para obtenerla.

A menudo se interpreta erróneamente que el derecho a la alimentación obliga al Estado a alimentar a su población, cuando de lo que se trata es de que el Estado -y particularmente el gobierno- respete y proteja el derecho de las personas a alimentarse. Para esclarecer las dudas, en 1999 el Comité de Derechos Económicos, Sociales y Culturales estableció en su
Observación general $N^{\circ} 12$ el ámbito de las obligaciones estatales al respecto, ${ }^{12}$ las que pueden clasificarse en tres niveles:

i) Obligación de respetar. Establece el límite al ejercicio del poder del Estado. Significa que el gobierno no debe interferir con los medios de subsistencia de las personas, ni dificultar su acceso a los alimentos o privarlas arbitrariamente de su derecho a ellos.

ii) Obligación de proteger. Significa que el gobierno debe promulgar leyes para evitar que personas $\mathrm{u}$ organizaciones poderosas conculquen el derecho a la alimentación, y también establecer órganos para investigar y proporcionar recursos eficaces si se viola ese derecho.

iii) Obligación de facilitar y en caso necesario proveer. Significa que el gobierno debe adoptar medidas positivas para individualizar a los grupos vulnerables y aplicar políticas para que tengan acceso a una alimentación suficiente, facilitando su capacidad de alimentarse. La obligación de proveer va más allá de la de facilitar, pero sólo aparece cuando la seguridad alimentaria de las personas se ve amenazada por motivos ajenos a su voluntad. Se considera que, como último recurso, puede ser necesaria la prestación de asistencia directa mediante redes de protección.

Estos tres niveles precisan mejor el ámbito de las obligaciones de los Estados en lo que se refiere a

\footnotetext{
12 Véase Naciones Unidas, Comité de Derechos Económicos Sociales y Culturales (1999).
} 
garantizar el derecho a la alimentación y sirven como un marco de referencia ética, política y legal. Basar políticas públicas de desarrollo rural en el ejercicio de los derechos humanos, particularmente el derecho a la alimentación, permite sacarlas del terreno de la caridad asistencial y hacerlas menos vulnerables a los vaivenes políticos. De esta forma se vuelven exigibles por la vía jurídica, sujetas a escrutinio y, eventualmente, a instancias de arbitraje.

A pesar de que el derecho a la alimentación se ha reconocido en diversos instrumentos internacionales, la preocupación por hacer que se le considere en su calidad de tal en las políticas de desarrollo rural y combate al hambre comenzó a cuajar ya entrado el decenio de 1990, en el marco del debate sobre los derechos de tercera generación. La Cumbre Mundial sobre la Alimentación de 1996 recogió este debate y estableció entre sus compromisos el de esclarecer el contenido del derecho a una alimentación suficiente y a no padecer hambre, junto con atender en especial a su aplicación.

Establecer una mejor definición del concepto del derecho a la alimentación, con miras a crear instrumentos concretos para mejorar su aplicación, ha comenzado a ser una preocupación importante de la sociedad civil y de algunos gobiernos del mundo. Al respecto, vale la pena mencionar la propuesta para crear un código de conducta internacional para el derecho a la alimentación. Aunque este código sería fundamentalmente un documento político más que legal, de aprobarse permitiría establecer una serie de obligaciones y responsabilidades a nivel nacional e internacional, con lo que se haría un valioso aporte a la construcción de una nueva institucionalidad mundial basada en el derecho a la alimentación y con fuerte influencia en el diseño de políticas de desarrollo.

\section{Descentralización y disparidades regionales}

A pesar de la aceptación casi universal de los méritos supuestos de las reformas de descentralización, y aunque éstas proliferaron ampliamente en la región, existe todavía un entendimiento científico bastante limitado de los arreglos institucionales que sustentan una gestión descentralizada efectiva. Además, un creciente cuerpo de evidencia empírica sugiere que la descentralización - administrativa, fiscal o política, o una combinación de ellas - puede facilitar el surgimiento de instituciones para la acción colectiva, pero no produce automáticamente las ventajas pensadas por sus partidarios. Por eso es necesario precaverse de ideas simplistas de que la sola descentralización resuelve algunos de los problemas más acuciantes del medio rural. Como en todo proceso de construcción institucional, lo que se requiere es una adecuada mezcla de intervenciones nacionales y locales. Aunque en general la descentralización ha sido vista como un mecanismo que fortalece la participación social en el diseño e implementación de políticas públicas, para evitar medidas aisladas que suelen dislocar la acción gubernamental quizás sea esencial insertar la descentralización en el marco de una política de desarrollo regional.

Más recientemente, producto de los propios procesos de globalización, se ha acrecentado la necesidad de promover diversas medidas enfocadas específicamente a disminuir las disparidades socioeconómicas entre territorios y ciudades y a optimizar sus oportunidades de desarrollo. Esas medidas se conocen ahora como políticas de desarrollo territorial (o más simplemente, como política territorial). Tales políticas no se enfocan sólo a los territorios más desfavorecidos, sino a todos, desde los más ricos hasta los más pobres. $\mathrm{Su}$ objetivo no es atraer inversiones a los territorios marginados por medio de subsidios y otros beneficios ofrecidos a los inversionistas, sino asegurar que todos sean capaces de maximizar sus oportunidades de desarrollo endógeno. Para alcanzar esta meta es clave aprovechar las ventajas de cada uno de ellos, el potencial de arrastre de sus ciudades y la creación de activos. No se trata desde luego de suspender las formas de asistencia y compensación a los territorios más pobres, que deben beneficiarse de una "ecualización financiera", sino de generar vínculos y enlaces adecuados entre aquellos que avanzan a un ritmo mayor y los que no lo hacen. Además, se debe asegurar infraestructura para todos, garantizándoles un mínimo nivel de accesibilidad. Puesto que esto depende de las características propias de cada uno de ellos, las políticas de infraestructura deben estar basadas en una tipología de territorios (Schejtman y Berdegué, 2003).

Los esquemas territoriales son por definición multisectoriales y sin duda deben ayudar a construir puentes entre los diversos objetivos de desarrollo, asî como a conciliarlos. Además, estos esquemas ofrecen dos beneficios adicionales: catalizan una mayor conciencia de la naturaleza y urgencia de los problemas, y ofrecen soluciones más compatibles con las restricciones locales (institucionales, financieras, ecológicas). Por lo tanto, cuando se obvia la heterogeneidad del sector rural, identificando el espacio rural con el espacio agropecuario, ${ }^{13}$ se reducen las potencialidades de

\footnotetext{
${ }^{13} \mathrm{Al}$ no tener en cuenta los nexos del sector rural con los núcleos urbanos, o la importancia del empleo rural no agrícola, cada vez más extendido y que condiciona cada vez más el desempeño rural.
} 
mejorar la competitividad y de mitigar la pobreza rural, pues no se consideran los factores que dinamizan la propia agricultura y que pueden derivarse del fortalecimiento de los vínculos con los núcleos urbanos inmediatos. El grueso de las actividades se centra entonces en lo agrícola, siendo que el diagnóstico debe considerar como espacio rural a aquel constituido por los núcleos urbanos a los que los pequeños productores están vinculados de manera directa.

Un examen de las demandas entre esos núcleos urbanos y el ámbito territorial de los pequeños productores agrícolas puede dar pistas sobre requerimientos que no están siendo satisfechos, o que lo están pero con altos costos de transacción. Con medidas simples, tales requerimientos podrían contribuir a elevar la competitividad de algunos de esos productores o, en el caso de familias con poca o ninguna tierra, mejorar sus condiciones de vida y de trabajo al reducir sus costos de transacción en una gama de actividades. En este sentido, para que los proyectos sean piezas clave de las estrategias de desarrollo rural es necesario que en su diseño se tengan en cuenta el territorio involucrado, los sectores de actividad en que se interviene, la estructura social del territorio, la duración de los procesos y una institucionalidad que asegure que sean sostenibles en el tiempo.

Las políticas de desarrollo territorial buscan elevar el valor agregado del territorio, lograr un crecimiento significativo de la economía rural y, en especial, fortalecer el capital social del territorio más que promover el desarrollo de una actividad económica en particular (Abramovay, 1999). Para esto, el desarrollo territorial incorpora una visión de cadenas productivas que va más allá de la agricultura ampliada, ${ }^{14}$ agregando la articulación de los distintos sectores y actores de una misma cadena.

\section{IV}

\section{La agricultura familiar}

\section{Un factor movilizador: los agricultores familia- res}

La experiencia de la última década, tanto en términos de movilización social como de esfuerzo productivo e innovaciones institucionales, nos dice que el segmento más dinámico del medio rural son los agricultores familiares, quienes en la región se caracterizan por la heterogeneidad de su dotación de recursos y de sus activos, es decir, de su capital natural, físico, financiero, humano y social (de Janvry y Sadoulet, 2001a). Esta desigualdad de condiciones más las fallas de mercado son algunas de las causas de la pobreza rural.

En una simplificación que, sin embargo, refleja las características principales del medio rural en América Latina y el Caribe, los agricultores familiares pueden clasificarse en dos grandes grupos, según el nivel de sus activos. En el primero están los campesinos cuyos recursos territoriales son tan reducidos que viven esencialmente como trabajadores asalariados - agrícolas o no agrícolas-, para los cuales la agricultura es un complemento. En el segundo se hallan los agricultores familiares, poseedores de tierras en diferentes cantidades, que obtienen gran parte de su ingreso de sus cultivos y lo complementan con la venta de su fuerza de trabajo (CEPAL, 1999).
En ambos grupos los trabajadores se ven obligados a recurrir a formas de empleo rural no agrícola que proveen gran parte del ingreso de los hogares rurales y son un sustituto de la tierra como fuente de ingresos. En un estudio recopilatorio ${ }^{15}$ efectuado por de Janvry y Sadoulet (2001a) se encontró que en el ejido mexicano, por ejemplo, el empleo rural no agrícola representaba el 55\% de los ingresos totales (de Janvry y Sadoulet, 2001b), en Nicaragua y Panamá el 61\% (Davis, Carletto y Sil, 1997; Banco Mundial, 1998), en Chile y El Salvador el 67\% y el 60\%, respectivamente (López y Valdés, 1997), y en Ecuador el 86\% (Lanjouw, 1996). Cabe añadir, sin embargo, que estas formas de complementar el ingreso no permiten a los agricultores familiares superar la condición de pobreza en que viven. Además, debido a fallas en las instituciones y mercados, en el último tiempo las familias dependen cada vez más del ingreso rural no agrícola

\footnotetext{
${ }^{14}$ La agricultura ampliada reconoce las distintas articulaciones que tiene la agricultura con otro tipo de actividades que se generan alrededor de ella. Estas actividades incluyen, entre otras, la demanda de insumos, de instrumentos y maquinarias, las actividades de poscosecha o de procesamiento de los productos agropecuarios, y los procesos de comercialización.

15 Basado en un trabajo pionero sobre las familias rurales en el sector reformado de México (Gordillo, de Janvry y Sadoulet (2000).
} 
(especialmente remesas) y de la inversión en animales y granos como fuente de ahorro y liquidez para casos de emergencia.

El proceso de transición desde una agricultura protegida a una con amplia base de crecimiento y competitividad necesitará mucho tiempo para acrecentar la baja inversión que predomina entre los agricultores familiares. La meta de mejorar el acceso a los alimentos está íntimamente ligada al mejoramiento de la competitividad de los agricultores familiares, sobre una base territorial y focalizada en la familia y en sus ingresos.

Pero si se sigue a Easterly (2002), se puede decir que la competitividad de los pequeños productores está también relacionada con el grado de inequidad que existe en el país, puesto que la desigualdad impide el desarrollo. Sokoloff y Engerman $(2002)^{16}$ plantean que la dotación de activos determina la inequidad, la cual a su vez determina la existencia de instituciones deficientes (poco democráticas e inestables), malas políticas de redistribución, baja inversión en capital humano y subdesarrollo.

Fortalecer el papel de los agricultores familiares está asociado a mejorar su competitividad en los mercados de tierras, productos, trabajo y financiamiento, para lo cual deben disponer de la información necesaria para insertarse en condiciones ventajosas en esos mercados. La hipótesis, según Escobal (2002), es que en las instituciones existen cuatro tipos de fallas que afectan la competitividad de las familias rurales: i) la asimetría de la información, ii) la disponibilidad y asignación de los bienes públicos, iii) las externalidades, y iv) los problemas de pobreza y equidad.

La asimetría de la información reduce el acceso a mercados necesarios para la competitividad de los pequeños productores. Hay problemas en el acceso a la información sobre los mecanismos de funcionamiento de los créditos rurales, tanto por parte de las familias rurales como por parte de los mismos bancos, y lo mismo sucede en el acceso a la propiedad de la tierra. También es muy importante que a las organizaciones de base ${ }^{17}$ se les facilite información sobre los programas y las políticas de desarrollo local, los mecanismos para acceder a fondos que financien proyectos y la forma de interactuar con el gobierno y el mercado (Alkire y otros, 2001).

16 Citados por Easterly (2002).

17 Los grupos con intereses comunes, como por ejemplo de mujeres, de ahorro, cooperativas, asociaciones de productores o de riego, y otros.
La escasez y mala asignación de bienes públicos dificulta el desarrollo de los sectores rurales. La dispersión espacial y la menor densidad de población guardan relación estrecha con infraestructuras inadecuadas y un acceso limitado a los servicios públicos, por lo cual las inversiones en estos sectores son consideradas de alto costo y riesgosas. Además, el acceso restringido a los bienes y servicios públicos debilita el mercado local de insumos y productos, lo que se suma a los altos costos de transacción para restringir las posibilidades de ahorro de las familias rurales. Es posible que los esfuerzos locales por organizarse colectivamente permitan avanzar en este aspecto, sobre todo cuando se trata de bienes y servicios de baja complejidad, ${ }^{18}$ en pequeña escala y que requieren de cooperación local, ya sea comunitarios (pastizales comunales, agua de riego y otros) o públicos (infraestructura local).

Los problemas de pobreza y desigualdad generan un círculo vicioso. ${ }^{19}$ Cuando las instituciones de mercado no resuelven estos problemas, los arreglos institucionales combinados de organismos públicos y organizaciones de la sociedad civil tienen que apoyar la equidad y sostenibilidad de las políticas y el consenso entre las partes. Para esto es necesario mejorar la estructura de la política social, a través de una gestión pública basada en la transparencia, el control ciudadano y la rendición de cuentas.

Por lo que se ha expuesto, se precisan políticas orientadas a elevar la competitividad de las familias rurales para que éstas puedan incrementar sus ingresos. Es fundamental mejorar la productividad de los más pobres y que esta mejora signifique su inserción en una economía en crecimiento, en el marco de mercados justos. Si las familias logran mejorar sus niveles de ingreso, tendrán seguridad alimentaria.

Como ya se ha señalado en diversos estudios, casi la mitad del ingreso percibido por las familias rurales en la región proviene de actividades no agropecuarias. Por lo demás, como consecuencia lógica de esta diversificación de las economías rurales, los procesos de inversión de capital en el sector rural son muy heterogéneos. En este sentido, las políticas destinadas

\footnotetext{
${ }^{18}$ Baja complejidad en este sentido se refiere a la homogeneidad de los actores y sus intereses.

${ }^{19}$ Lynn Karl (2002) ha explicado este círculo vicioso como una situación en la que la pobreza y los altos niveles de desigualdad se reproducen mutuamente y frenan a su vez el crecimiento económico. Dicha situación disminuye apreciablemente la capacidad para atender los problemas derivados de la pobreza y la inequidad.
} 
a incrementar la competitividad de las familias rurales deben considerar la heterogeneidad y diversificación de los mercados rurales, ${ }^{20} \mathrm{y}$ apuntar a un desarrollo territorial más que sectorial.

En la agricultura, la heterogeneidad implica la coexistencia de dos sectores en cuyo interior hay también diversas modalidades. De esos dos sectores, uno es moderno, comercial, hace uso intensivo de capital y tecnología y se orienta principalmente a las exportaciones. El otro hace uso intensivo de mano de obra de baja productividad, exhibe grandes deficiencias y segmentaciones, tiene subdotación de activos y muy baja inversión, todo lo cual dificulta el logro de la eficiencia, competitividad, reconversión y rentabilidad que una política sectorial puede buscar. La experiencia ha mostrado que la mayor parte de las familias rurales en situación de riesgo alimentario depende de este tipo de agricultura. Por su parte, el sector agrícola moderno, más que crear demandas importantes de mano de obra, ha desplazado población y ha absorbido la mayor parte de la ayuda a la agricultura, debido a su mayor capacidad de negociación y su poder político. Con esto ha acentuado la concentración de la tierra y los recursos productivos, y la brecha entre la agricultura comercial y la agricultura familiar

Los agricultores familiares, por lo tanto, encaran restricciones internas y externas para mejorar su competitividad. Una de las formas para enfrentarlas es buscar el incremento de su capital social y humano, acrecentando su participación en organizaciones que les permitan influir en el diseño y aplicación de políticas de desarrollo y comercialización, y también mejorando sus técnicas de producción. A su vez, los gobiernos debieran contribuir a mejorar el funcionamiento y la confiabilidad de los mercados de productos, tierras, trabajo y financiamiento, así como la confianza de los productores en los dispositivos de seguridad social para superar limitaciones en materia de recursos naturales, mercado e infraestructura. Además, los Estados debieran tratar de mitigar la inequidad a través de instituciones más democráticas y estables, mejores políticas de redistribución y una mayor inversión en capital humano.

\footnotetext{
${ }^{20}$ En el mundo rural se ha dado un vigoroso proceso de crecimiento de los sectores de servicios no ligados directamente a las cadenas agroalimentarias e industriales, sino a la demanda de los asentamientos humanos rurales. Además, las actividades de construcción, infraestructura y servicios públicos aparecen cada vez más dentro del marco de la economía rural, restando participación al sector agropecuario.
}

\section{Un programa de apoyo a la agricultura familiar}

Con base en lo anterior, se ha estilizado ${ }^{21}$ un programa mínimo para el fomento de la agricultura y el desarrollo rural, que combina intervenciones de políticas de desarrollo encaminadas a mejorar el funcionamiento de los mercados rurales. Se partió constatando que la dispersión de instrumentos daña a los actores rurales, afecta especialmente a los agricultores familiares, genera mayores costos de transacción, propicia la corrupción y promueve disputas entre las burocracias estatales, originando duplicaciones de esfuerzo. Por ello nunca se insistirá lo suficiente en que, más allá del monto de los recursos públicos que se destinen al medio rural, importa la forma en que ellos se canalizan.

Dicho programa mínimo debe contener algunos elementos que permitan generar una estrategia básica para impulsar nuevas formas públicas de apoyo al sector rural. Sus objetivos son los siguientes:

i) Hacer más competitiva la producción de los habitantes rurales afectados por los cambios económicos y las reformas estructurales en la región, mediante apoyos directos, temporales y selectivos que los pongan en condiciones de competir en nuevas áreas de especialización, con pleno respeto a la conservación de los recursos naturales. Una política de ingresos rurales, cuya continuidad sea garantizada por la ley y periódicamente revisable, ${ }^{22}$ puede convertirse en el núcleo de un programa unificado de apoyo a la agricultura familiar.

ii) Promover acciones de asociación voluntaria y autosostenible de los grupos rurales afectados, a fin de que puedan formar organizaciones capaces de mantener en el tiempo los logros iniciales de estos programas de apoyo a los ingresos.

iii) Proporcionar asistencia directa y servicios a los hogares en áreas desfavorecidas. Esta política de apoyo directo puede convertirse en el punto de partida para un conjunto de instrumentos de política que, adecuadamente diseñados, contribuyan a fortalecer los nuevos vínculos entre las políticas, los productores y otros agentes económicos.

\footnotetext{
${ }^{21}$ Es decir, se ha planteado un conjunto de elementos invariables que evidentemente deben adaptarse al carácter muy heterogéneo del medio rural de la región, y a prioridades que dependen también de cada contexto.

${ }^{22}$ Un ejemplo de lo anterior es el Programa de Apoyos Directos al Campo (PROCAMPO) en México, que puede sentar las bases para esto particularmente con las recientes modificaciones introducidas en la Ley de Capitalización Rural.
} 
iv) Un sistema de financiamiento rural que movilice el ahorro en el marco de la agricultura familiar y haga hincapié en la formación de capital.

v) Una política de infraestructura que, sin olvidar algunos grandes proyectos estratégicos de riego, se oriente a aumentar considerablemente tanto la pequeña infraestructura productiva (riego, conservación de suelos y de acuíferos) como la infraestructura comercial (caminos, bodegas, frigoríficos y sistemas de transporte).

vi) Una política de fomento de la innovación y la transferencia tecnológica que implique la integración de universidades e institutos tecnológicos en un programa de divulgación y transferencia masiva de habilidades y conocimientos, apoyado por nuevas interacciones con los productores y teniendo como respaldo la premisa de que el capital humano es un factor fundamental de la competitividad.

vii) Políticas equitativas y sostenibles de acceso a los recursos naturales necesarios para sostener medios de vida adecuados a las poblaciones rurales de la región, incluyendo entre esas políticas, según sea el caso, reformas agrarias y otros mecanismos de acceso a la tierra.
Abramovay, R. (1999): Sete desafíos para desenvolvimento territorial, Fundação Lyndolpho Silva/Banco Nacional da Agricultura Familiar (BNAF), www.bnaf.org.br/palest04.htm.

Alkire, S. y otros (2001): Community-driven development, PRSP Sourcebook, www.worldbank.org/poverty/strategies/ sourctoc.htm.

Banco Mundial (1998): Panama LSMS, Washington, D.C.

Burfisher, M.E. (comp.) (2001): Agricultural Policy Reform in the WTO: The Road Ahead, ERS Agricultural Economics Report, $\mathrm{N}^{\mathrm{o}}$ 802, Washington, D.C., Departamento de Agricultura de los Estados Unidos.

CEPAL (Comisión Económica para América Latina y el Caribe) (1999): Efectos sociales de la globalización sobre la economía campesina: reflexiones a partir de experiencias en México, Honduras y Nicaragua, LC/MEX/L.382, México, D.F., Sede Subregional de la CEPAL en México.

(2002): Panorama social de América Latina, 2001-2002, LC/G.2183-P, Santiago de Chile, octubre. Publicación de las Naciones Unidas, $\mathrm{N}^{\circ}$ de venta: S.02.II.G.65.

Davis, B., C. Carletto y J. Sil (1997): Los hogares agropecuarios en Nicaragua: un análisis de tipología, Berkeley, Universidad de California.

De Janvry, A. y E. Sadoulet (2001a): La inversión en el desarrollo rural es buen negocio, en R. Echeverría (comp.), Desarrollo de las economías rurales en América Latina y el Caribe, Washington, D.C., Banco Interamericano de Desarrollo (BID).

(2001b): Income strategies among rural households in Mexico: The role of off-farm activities, World Development, vol. $29, \mathrm{~N}^{\circ} 3$, Amsterdam, Elsevier Science.

Easterly, W. (2002): Inequality Does Cause Underdevelopment: New Evidence, Washington, D.C., Center for Global Development.

Escobal, J. (2002): Nuevas instituciones para el desarrollo agrícola y rural en América Latina y el Caribe, CUREMIS II, Roma, Organización de las Naciones Unidas para la Agricultura y la Alimentación (FAO).

FAO (Organización de las Naciones Unidas para la Agricultura y la Alimentación) (2000): El estado mundial de la agricultura y la alimentación, 2000, Roma.

(2002a): El estado de la inseguridad alimentaria en el mundo, 2002, Roma.

(2002b): Cumbre Mundial sobre la Alimentación: cinco años después, www.fao.org/worldfoodsummmitt/spanish/ index.htm

(2002c): Evaluación externa independiente del Programa Especial para la Seguridad Alimentaria (PESA), Roma, www.fao.org/spfs/ y www.fao.org/docrep/meeting/004/y672s/ y6172s99.htm.

Gordillo, G. (1999): La ansiedad por concluir: lecciones de las reformas estructurales en América Latina. Mercado de valores, México, D.F., Nacional Financiera, octubre. También disponible en www.rlc.fao.org/quiensom/rlc/gordillo/ default.htm.

(2003): El desarrollo rural y el combate al hambre, la pobreza y la desigualdad, documento presentado en el Taller "La importancia del enfoque territorial en el desarrollo rural de América Latina y el Caribe" (Milán, 20 y 21 de marzo de 2003), organizado por el Fondo Internacional de Desarrollo Agrícola (FIDA), la Organización de las Naciones Unidas para la Agricultura y la Alimentación (FAO) y el Banco Interamericano de Desarrollo (BID), con el financiamiento del gobierno de Italia.

Gordillo, G., A. de Janvry y E. Sadoulet (2000): La segunda reforma agraria en México, México, D.F., Fondo de Cultura Económica.

Hirschman, A. (1963): Journeys towards Progress, Nueva York, Twentieth Century Fund.

Izam, M. y V. Onffroy (2000): El sector agrícola en la integración económica regional: experiencias comparadas de América Latina y la Unión Europea, serie Comercio internacional, $\mathrm{N}^{\circ}$ 8, LC/L.1419, Santiago de Chile, Comisión Económica para América Latina y el Caribe (CEPAL). Publicación de las Naciones Unidas, $\mathrm{N}^{\mathrm{o}}$ de venta: S.00.II.G.91.

Karl, T.L. (2002): The Vicious Cycle of Inequality in Latin America, Working Paper, No 2002-177, Berkeley, Universidad de California.

Lanjouw, P. (1996): Rural Poverty and Non Agricultural Employment in Ecuador, Washington, D.C., Departamento de Investigaciones sobre Políticas de Desarrollo, Banco Mundial.

López, R. y A. Valdés (1997): Rural Poverty in Latin America: Analytics, New Empirical Evidence and Policy, Washington, D.C., Departamento Técnico, Banco Mundial.

Naciones Unidas, Comité de Derechos Económicos, Sociales y Culturales (1999): Cuestiones sustantivas que se plantean en la aplicación del Pacto Internacional de Derechos Económicos, Sociales y Culturales, Observación general $\mathrm{N}^{\circ} 12,20^{\circ}$ período de sesiones del Comité, Ginebra, 26 de abril.

Polanyi, K. (1957): The Great Transformation: The Political and Economic Origins of Our Time, Boston, Beacon Press.

Rello, F. y Y. Trápaga (2001): Libre mercado y agricultura: efectos de la Ronda Uruguay en Costa Rica y México, LC/L.1668-P, México, D.F., Sede Subregional de la CEPAL en México. 
Publicación de las Naciones Unidas, $\mathrm{N}^{\circ}$ de venta: S.01.II.G.203.

Schejtman, A. y J. Berdegué (2003): Desarrollo territorial rural, Santiago de Chile, Red Internacional de Metodología de Investigación de Sistemas de Producción (RIMISP).

Sokoloff, K. y S. Engerman (2002): Factor Endowments, Inequality, And Paths Of Development Among New World
Economies, NBER Working Paper, No 9259, Cambridge, Massachusetts, National Bureau of Economic Research, octubre.

Székely, M. y J. Londoño (1998): Sorpresas distributivas después de una década de reformas: Latinoamérica en los noventa, Pensamiento iberoamericano, vol. extraordinario, Madrid, Universidad Complutense de Madrid. 